\title{
Properties of the invariant disk packing in a model bandpass sigma-delta modulator
}

\author{
Peter Ashwin, Xin-Chu Fu \\ School of Mathematical Sciences \\ Laver Building \\ University of Exeter \\ Exeter EX4 4QE, U. K. \\ Jonathan Deane \\ Department of Mathematics and Statistics \\ School of Electronics, IT and Mathematics \\ University of Surrey \\ Guildford GU2 7XH, U. K. \\ P.Ashwin, Fu-Xin-Chu@ex.ac.uk, J.Deane@eim.surrey.ac.uk
}

January 7, 2002

\begin{abstract}
In this paper we discuss the packing properties of invariant disks defined by periodic behaviour of a model for a bandpass $\Sigma-\Delta$ modulator. The periodically coded regions form a packing of the forward invariant phase space by invariant disks. For this oneparameter family of PWIs, by introducing codings underlying the map operations we give explicit expressions for the centres of the disks by analytic functions of the parameters, and then show that tangencies between disks in the packings are very rare; more precisely they occur on parameter values that are at most countably infinite. We indicate how similar results can be obtained for other plane maps that are piecewise isometries.
\end{abstract}

Key words: Piecewise isometry, discontinuous dynamical system, symbolic coding, disk packing, sigma-delta modulator.

\section{Introduction}

We study some dynamical properties of a particular map modelling the quiescent response of a second order bandpass $\Sigma-\Delta$ modulator of Feely et al. $[9,10]$ in the case of unity gain. This is a one-parameter planar map that can be shown [4] to be equivalent to a family of piecewise isometries $f_{\theta}$ on a global attractor consisting of two trapezia over a certain range of the parameter $\theta$.

A planar piecewise isometry (PWI) is a piecewise continuous map that is an isometry on each of a number of polygonal pieces (called atoms) on the plane. Although PWIs are geometrically very simple, their dynamical behaviours under iteration can be very rich; see for example, $[1,17,13,14,8]$ and there remain many fundamental questions for such maps that are unanswered. 
For piecewise isometries there is a natural coding given by the itinerary of a trajectory between the atoms. A subset of points with the same coding is referred to as a cell [13]. The periodically coded cells define an invariant set that is a disjoint union of disks if none of the rotations are rational. This union is referred to as the invariant disk packing for the PWI; we describe this more precisely in section 2.3.

One question of concern is whether the complement of the disk packing, or the subset with aperiodic coding possesses positive measure. Such a result would imply that the map can undergo aperiodic behaviour with positive probability for a randomly chosen initial condition; we conjecture that this is typically the case.

Although a recent example in [5] shows that a tangent-free dense disk packing may have zero measure complement, the presence of a "loose" disk packing (e.g., one with rare tangencies between disks) would support the positive measure complement conjecture. Numerical approximation [3] of a disk packing for a typical parameter of the overflow oscillation map supports the conjecture that the measure of the complement is positive.

The main result in this paper is Theorem 1, which shows that there are no tangencies in the disk packing of the $\Sigma-\Delta$ map for almost all values of $\theta$, generalizing the result of [5] to this new family of maps. For this one-parameter family of PWIs, by introducing codings underlying the map operations, we give explicit expressions for the centres of the disks by analytic functions of the parameters, and then show that tangencies between disks in the packings are very rare; more precisely they occur on parameter values that are at most countably infinite and hence zero measure. We show how this result can be adapted for other maps, namely the Goetz map, and discuss obstructions to generalizing this approach to more general PWIs.

We discuss briefly some other piecewise isometries such as a planar area preserving map derived from a kicked Hamiltonian system and the Goetz map, showing similarly that the periodically coded regions forming a packing of the phase space by invariant disks also have typically no tangents.

\section{The bandpass $\Sigma-\Delta$ modulator as invertible piecewise isometries on two trapezia}

We consider the iterated mapping

$$
w_{n+2}=2 r \cos \theta w_{n+1}-r^{2} w_{n}+2 r \cos \theta\left(x_{n+1}-\operatorname{sgn} w_{n+1}\right)-r^{2}\left(x_{n}-\operatorname{sgn} w_{n}\right)
$$

introduced in Feely et al $[10,9,11]$, and discussed in $[12,4]$. For unity gain, $r=1$, the feedback loop of the modulator contains a filter with transfer function

$$
F(z)=\frac{2 z \cos \theta-1}{z^{2}-2 z \cos \theta+1}
$$

where $\theta$ is a parameter that sets the centre frequency of the passband. The output from the modulator is the quantization $\operatorname{sgn}\left(w_{n}\right)$ given the input $x_{n}$. We are interested in the quiescent behaviour, $x_{n} \equiv 0$, with unity gain, and so we re-write the mapping in vector form as

$$
\left(\begin{array}{l}
w_{n+1} \\
y_{n+1}
\end{array}\right)=\left(\begin{array}{cc}
0 & 1 \\
-1 & 2 \cos \theta
\end{array}\right)\left(\begin{array}{c}
w_{n} \\
y_{n}
\end{array}\right)+\left(\begin{array}{c}
0 \\
\operatorname{sgn} w_{n}-2 \cos \theta \operatorname{sgn} y_{n}
\end{array}\right) .
$$



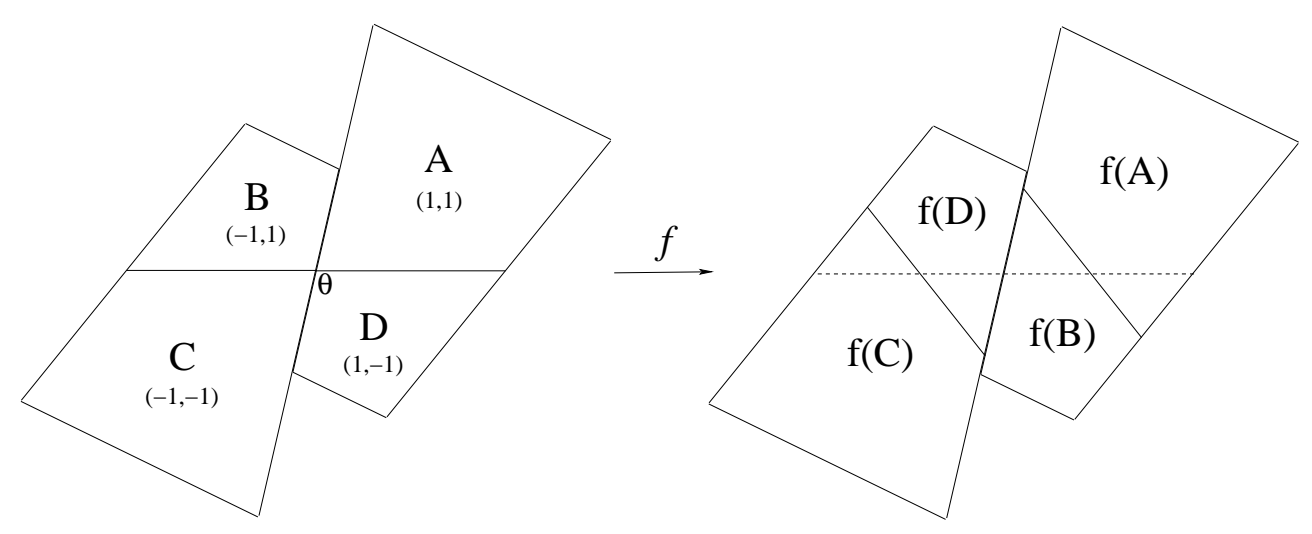

Figure 1: The two trapezia on which the piecewise isometry is defined. There is a oneparameter of such maps parameterized by the angle $\theta$, where $\pi / 3 \leq \theta \leq 2 \pi / 3$ or $4 \pi / 3 \leq$ $\theta \leq 5 \pi / 3$.

The eigenvalues of the matrix are $\mathrm{e}^{ \pm i \theta}$. The first part of the transformation therefore preserves area and so can be conjugated to a solid rotation by shearing the coordinates. The mapping is invertible on a set $M_{\theta}$ consisting of two trapezia.

To see this we define $u_{n}=w_{n} / \sin \theta-y_{n} \cot \theta, v_{n}=y_{n}$, and the state vector $\mathbf{x}_{n}=$ $\left[u_{n}, v_{n}\right]^{T}$. With this transformation (2) can be written in Jordan normal form as

$$
\mathbf{x}_{n+1}=f\left(\mathbf{x}_{n}\right)=R \mathbf{x}_{n}+\sigma\left(\mathbf{x}_{n}\right)\left(\begin{array}{c}
-\cot \theta \\
1
\end{array}\right)
$$

where $\sigma\left(\mathbf{x}_{n}\right)=\operatorname{sgn}\left(u_{n} \sin \theta+v_{n} \cos \theta\right)-2 \cos \theta \operatorname{sgn} v_{n}$ and $R$ represents a pure clockwise rotation by $\theta$. Note that given any trajectory $\mathbf{x}_{n}$ this will define a binary sequence given by the quantizer output $\sigma\left(\mathbf{x}_{n}\right)$.

Fix $\theta$ such that $\pi / 3 \leq \theta \leq 2 \pi / 3$ or $4 \pi / 3 \leq \theta \leq 5 \pi / 3$, and consider the two trapezia $M_{\theta}$ :

$$
M_{\theta}=A \cup B \cup C \cup D,
$$

illustrated in Figure 1. Note that $f$ is actually defined on the whole plane, but $M_{\theta}$ is the maximal invariant set of $f$, and $M_{\theta}$ is the global attractor for all initial conditions in the plane.

\section{$2.1 \quad$ Numerical simulations}

To more clearly illustrate the disk packing, we provide here some numerical simulations. Figure 2 was calculated by iterating a number of randomly chosen initial conditions; one can clearly see an apparent packing of the global attractor by quasiperiodic behaviour. To examine this packing in more detail, Figures 3 and 4 show some invariant discs for reasonably short periods in the complex plane $z=\operatorname{Re}(z)+i \operatorname{Im}(z)$. These were calculated numerically as follows. At stage $i$, assume that the centres and radii of $\operatorname{discs} D_{1} \ldots D_{i}$ have been found.

1. Generate a random point $z \in M_{\theta} \backslash \bigcup_{j=1}^{i} D_{j}$ 


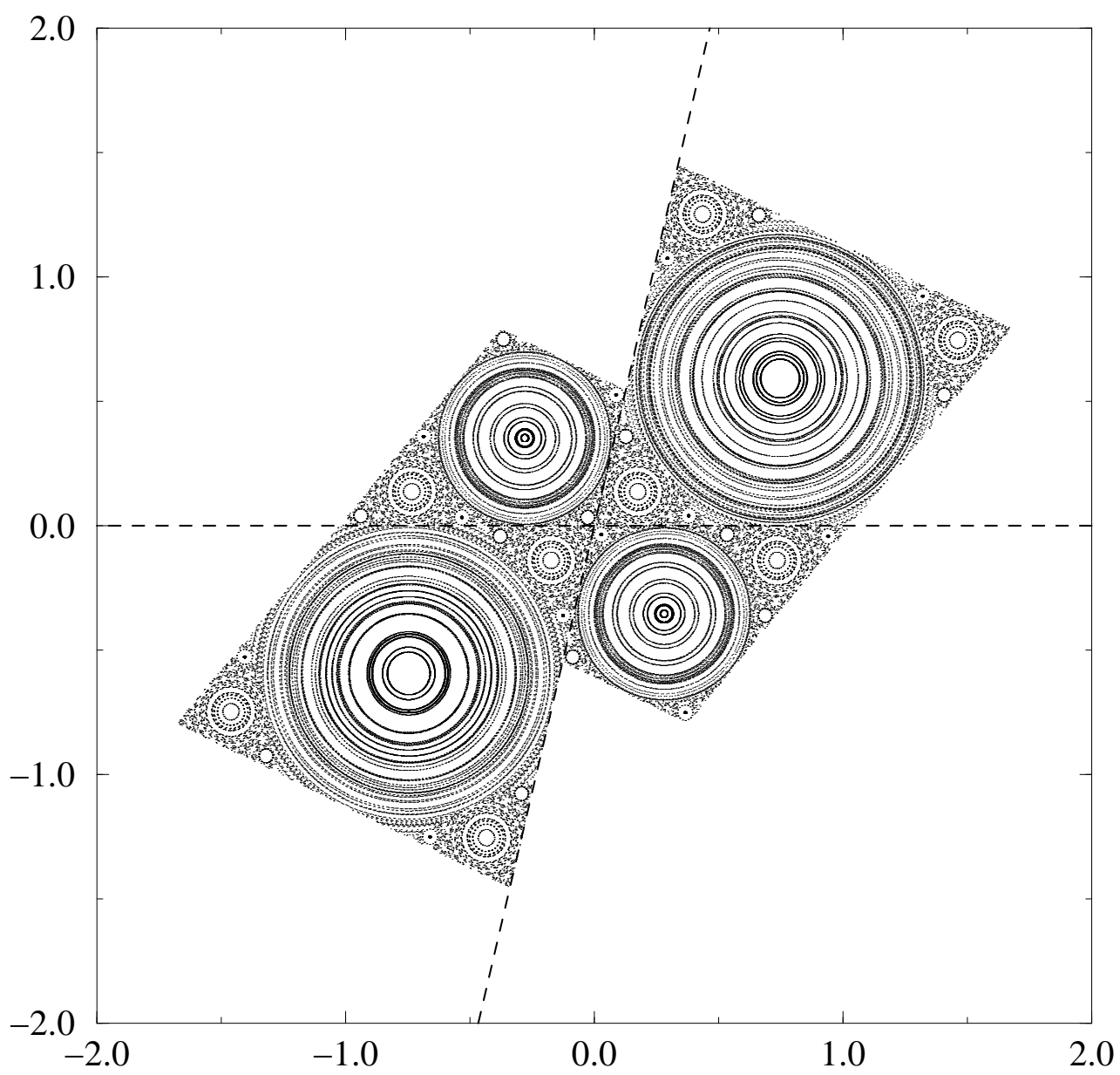

Figure 2: Some orbits of the iterated $\Sigma-\Delta$ map (3) when $\theta=1.8$ radians. The diagram was generated by choosing 150 random initial conditions within $M_{\theta}$ and plotting 500 iterates, starting from each of these initial points. All other initial conditions are observed to enter $M_{\theta}$ after a finite number of iterates. 


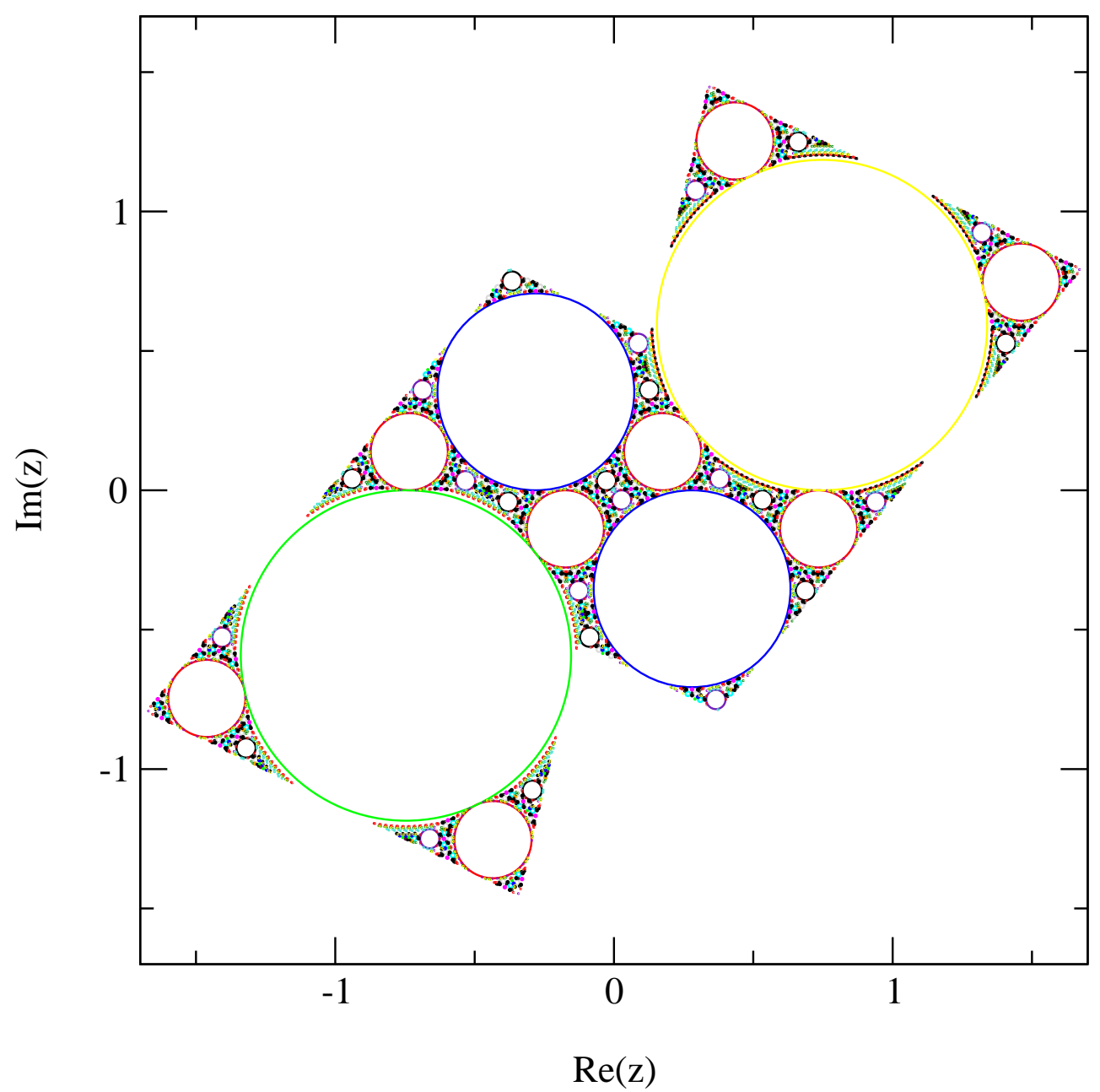

Figure 3: The invariant disk packing for the $\Sigma-\Delta$ map (3) with $\theta=1.8$ radians. Different colours refer to the boundaries of disks with different periodic codings. Theorem 1 indicates that the invariant disk packing has rare tangencies between disks and in this sense is a 'loose' packing for almost all values of $\theta$. 


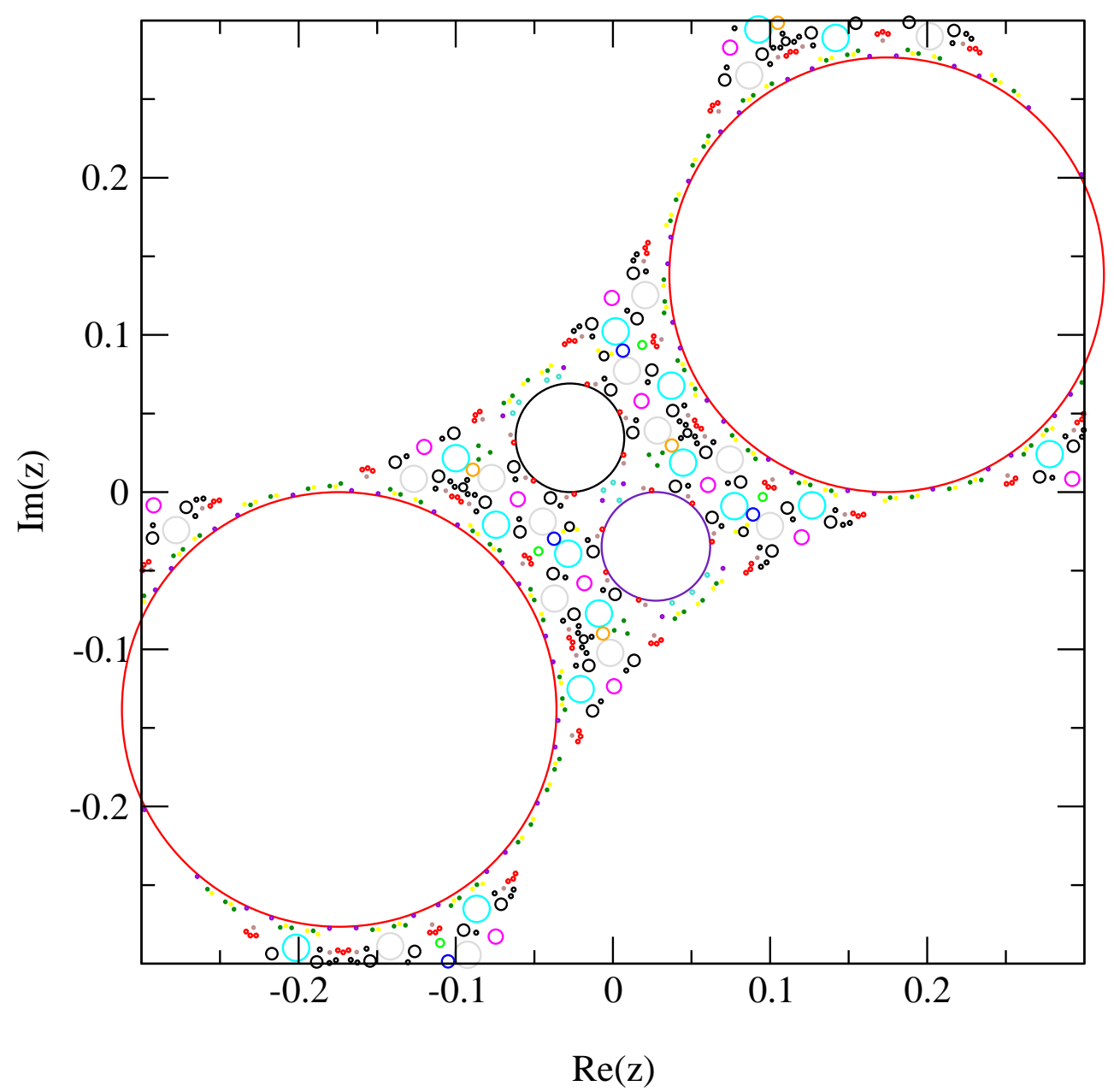

Figure 4: Zoom into a detail of Figure 3. Only disk boundaries that are centred within the axes are included. Note that any apparent tangencies can be seen to be absent on closer inspection. 
2. Generate the sequence $z, f(z), f^{2}(z), \ldots, f^{N}(z)$ for some large $N$.

3. Check for periodicity $\leq M$ with $M \ll N$. (In our calculations, $N=500000, M=$ 4000.)

4. If the sequence is periodic with period $P$, estimate (A) the centres and (B) the radii of the $P \operatorname{discs} D_{i+1} \ldots D_{i+P}$ with this period.

5. Return to 1 .

Calculations (A) and (B) are carried out as follows.

(A) The position of the centre of a given disc is estimated from the approximately $N / P$ points within the disc, by choosing three of these points which are as near as possible to being angularly separated by $2 \pi / 3$ radians. This minimizes the error in the centre calculation, compared to using points that happen to lie within a very small arc. Simple co-ordinate geometry then allows the calculation of the centre of this disc, $z_{0}$ say, and gives a lower bound on its radius, $r_{0}$ say. The error in the centre estimate is then easily found by calculating $\left|z_{0}-f^{P}\left(z_{0}\right)\right|$.

(B) We now have a lower bound on the radius $r_{0}$ of the disc. An upper bound is found by gradually increasing the radius, in practice by successively multiplying by a constant factor $\rho$, until iteration starting from a point on the circumference of this enlarged disc no longer results in period- $P$ behaviour. The radius of the disc is now bracketed between the current radius and the current radius/ $\rho$. Finally, a bisection method is used to obtain a more accurate approximation to the radius of the disc. An error estimate on this is the final lower and upper bounds obtained by the bisection algorithm. In our calculations, we used $\rho=1.1$. All discs are treated separately, even though each disc on an orbit of a given period must have the same radius.

\subsection{Coding the trajectories}

We rewrite $f: M_{\theta} \rightarrow M_{\theta}$ in complex form as

$$
f(z)=e^{-i \theta} z+W \sigma(z)
$$

where $W=-\frac{\omega}{\sin \theta}, \omega=e^{-i \theta}$, and

$$
\sigma(z)=\left\{\begin{aligned}
1-2 \cos \theta & \text { if } z \in A \\
-1-2 \cos \theta & \text { if } z \in B \\
-1+2 \cos \theta & \text { if } z \in C \\
1+2 \cos \theta & \text { if } z \in D
\end{aligned}\right.
$$

Assign each $A, B, C, D$ with a pair $k(z)=\left(k^{\prime}(z), k^{\prime \prime}(z)\right)$ where

$$
\left(k^{\prime}(z), k^{\prime \prime}(z)\right)=\left\{\begin{array}{cc}
(1,1) & \text { for } z \in A \\
(-1,1) & \text { for } z \in B \\
(-1,-1) & \text { for } z \in C \\
(1,-1) & \text { for } z \in D
\end{array}\right.
$$

Then $f$ can be written as

$$
f(z)=\omega z+W k^{\prime}(z)-2 \cos \theta W k^{\prime \prime}(z) .
$$


Obviously, $k(z)=\left(k^{\prime}(z), k^{\prime \prime}(z)\right)$ identifies the partition elements $A, B, C, D$ and we use this coding.

There is a full measure subset of $x \in M_{\theta}$ such that we can define a unique itinerary; this is a map $\iota: M_{\theta} \rightarrow \Sigma(4)$ with $\Sigma(4)$ the set of bi-infinite words with 4 letters, i.e., the four pairs $(1,1),(-1,1),(-1,-1),(1,-1)$. These signs simply correspond to the output of the quantizer for the current state of the system, and the output for the next state.

We say $\mathbf{s}=\left(s_{0}, s_{1}, s_{2}, \ldots s_{j} \ldots\right) \in \Sigma(4)$ is admissible if there is an $x \in M_{\theta}$ such that $\iota(x)=\mathbf{s}$. A sequence $\mathbf{s}$ is periodic if there is a smallest $N>0$ such that $s_{k+N}=s_{k}$ for all $k ; N$ is the period. The set of points with the same itinerary we refer to as a cell, i.e. given an admissible $\mathbf{s}$ we define $C(\mathbf{s})=\iota^{-1}(\mathbf{s})$. We write $\mathcal{P} \subset \Sigma(4)$ to be the set of admissible periodic itineraries. By convexity of the atoms and the preservation of this under isometries it is routine to show that the cells $C(\mathbf{s})$ are convex planar regions; these have interior if and only if $\mathbf{s} \in \mathcal{P}$. For all admissible $\mathbf{s} \notin \mathcal{P}$ the cell is either a point or a line segment $([18,2,14,15])$. This is because the orbit of an aperiodic sequence will have an infinite number of cells; if these have finite area, their total area is infinite, giving a contradiction.

If an orbit has itinerary $\mathbf{k}=\left(k_{0} k_{1} k_{2} \cdots k_{j} \cdots\right)$, where

$$
k_{j}=\left(k_{j}^{\prime}, k_{j}^{\prime \prime}\right) \in\{(1,1),(-1,1),(-1,-1),(1,-1)\}
$$

the $n$-th iteration can be expressed as

$$
f^{n}(z)=\omega^{n} z+W \sum_{j=0}^{n-1} \omega^{j} k_{n-j-1}^{\prime}-2 \cos \theta W \sum_{j=0}^{n-1} \omega^{j} k_{n-j-1}^{\prime \prime}
$$

Consider any periodic itinerary $\mathbf{k}$ of minimal period $n$ such that

$$
\mathbf{k}=P\left(k_{0} k_{1} k_{2} \cdots k_{n-1}\right)
$$

where $P$ denotes the periodic concatenation of its argument. If this is admissible then there will be a unique periodic point $z=z_{\mathbf{k}}$ with $\iota(z)=\mathbf{k}$ satisfying

$$
z_{\mathbf{k}}=\frac{W}{1-\omega^{n}}\left(\sum_{j=0}^{n-1} \omega^{j} k_{n-j-1}^{\prime}-2 \cos \theta \sum_{j=0}^{n-1} \omega^{j} k_{n-j-1}^{\prime \prime}\right) .
$$

Letting

$$
\theta=-2 \tau \text { and } \eta=e^{i \tau}
$$

this can be written as

$$
z_{\mathbf{k}}=\frac{i}{2 \sin 2 \tau \sin n \tau}\left(\sum_{j=0}^{n-1} \eta^{n-2 j} k_{j}^{\prime}-2 \cos 2 \tau \sum_{j=0}^{n-1} \eta^{n-2 j} k_{j}^{\prime \prime}\right) .
$$

\subsection{Genericity of tangent-free disk packings for the family of $\Sigma-\Delta$ maps}

We say a PWI is an irrational rearrangement if $\left.f\right|_{M_{k}}(x)=e^{i \theta} x+w_{k}$ and $\theta / \pi \notin \mathbb{Q}$, where $M_{k}$ is the $k$-th partition of $M_{\theta}$. Any $n$-periodic cell for an irrational rearrangement is a disk. The $n^{\text {th }}$ iterate of the map acts as an irrational rotation on the disk, i.e. its centre is an $n$-periodic point and it is surrounded by a nested set of invariant circles $[13,15]$. Hence, 
for PWIs that are irrational rearrangements, the set of periodic cells defines a union of disjoint disks

$$
\mathcal{C}=\bigcup_{\mathbf{s} \in \mathcal{P}} C(\mathbf{s})
$$

that we refer to as the invariant disk packing of $M$ induced by the piecewise isometry.

As a piecewise isometry, the $\Sigma-\Delta$ modulator $f$ is an irrational rearrangement when $\theta / \pi \notin \mathbb{Q}$. So there is an invariant disk packing of $M_{\theta}$ composed of periodic and quasiperiodic solutions if $\theta / \pi$ is irrational (see Figure 3 for a numerical approximation to this for the $\Sigma-\Delta$ modulator with $\theta=1.8$ ). For rational $\theta / \pi$ there is invariant polygon packing of $M_{\theta}$ composed only periodic solutions. The complement of this invariant disk/polygon packing (which may have positive measure) possesses orbits whose codings are all aperiodic and may display complicated dynamical behaviour.

The numerical observations (see Figures 3 and 4 ) indicate that the invariant disk packing induced by the $\Sigma-\Delta$ modulator has no tangencies and in this sense is a 'loose' packing. Although we cannot prove this for any given value of $\theta$, we can prove that tangencies are extremely rare within this one parameter family of $\Sigma-\Delta$ modulator maps. We first prove a lemma:

Lemma 1 Suppose that $\mathbf{k}, \mathbf{l} \in \mathcal{P}$ are nonidentical admissible periodic sequences. Then the largest inscribed disks of these cells with these codings are tangent for at most a finite number of values of $\theta$. In consequence, there are only finitely many irrational $\theta$ at which they can be tangent.

Proof Suppose that the two disks $C(\mathbf{k})$ and $C(\mathbf{l})$ are tangent. If they are in the same atom, then their images under $f$ will still be tangent. Hence we can assume without loss of generality (by iterating if necessary) that $C(\mathbf{k})$ and $C(\mathbf{l})$ are in different atoms.

The tangencies must occur at the tangencies of the atoms. Note that if centres of the disks are given by $z_{\mathbf{k}}$ and $z_{1}$ whenever there is a tangency then one of the two cases below must hold between tangent disks:

\begin{tabular}{c|cc} 
& Case & Condition \\
\hline$I$ & $\left(z_{\mathbf{k}} \in A\right.$ and $\left.z_{\mathbf{l}} \in D\right)$ & $\operatorname{Re}\left(z_{\mathbf{k}}-z_{\mathbf{l}}\right)=0$ \\
& or $\left(z_{\mathbf{k}} \in B\right.$ and $\left.z_{\mathbf{l}} \in C\right)$ & \\
\hline$I I$ & $\left(z_{\mathbf{k}} \in A\right.$ and $\left.z_{\mathbf{l}} \in B\right)$ & $\operatorname{Re}\left(e^{-2 i \tau}\left(z_{\mathbf{k}}-z_{\mathbf{l}}\right)\right)=0$. \\
& or $\left(z_{\mathbf{k}} \in C\right.$ and $\left.z_{\mathbf{l}} \in D\right)$ &
\end{tabular}

Let $\mathbf{k}=P\left(k_{0} k_{1} \cdots k_{n-1}\right)$ and $\mathbf{l}=P\left(l_{0} l_{1} \cdots l_{m-1}\right)$. Then using $(6)$ we have

$$
\begin{aligned}
z_{\mathbf{k}}-z_{\mathbf{l}}= & \frac{i}{2 \sin 2 \tau \sin n \tau}\left(\sum_{j=0}^{n-1} \eta^{n-2 j} k_{j}^{\prime}-2 \cos 2 \tau \sum_{j=0}^{n-1} \eta^{n-2 j} k_{j}^{\prime \prime}\right) \\
- & \frac{i}{2 \sin 2 \tau \sin m \tau}\left(\sum_{j=0}^{m-1} \eta^{m-2 j} l_{j}^{\prime}-2 \cos 2 \tau \sum_{j=0}^{m-1} \eta^{m-2 j} l_{j}^{\prime \prime}\right) .
\end{aligned}
$$

Let $\phi_{1}(\tau)=-\sin 2 \tau \operatorname{Re}\left(z_{\mathbf{k}}-z_{\mathbf{l}}\right), \phi_{2}(\tau)=-\sin 2 \tau \operatorname{Re}\left((\cos 2 \tau-i \sin 2 \tau)\left(z_{\mathbf{k}}-z_{\mathbf{l}}\right)\right)$, then we 
can calculate

$$
\begin{aligned}
\phi_{1}(\tau) & =\frac{1}{2 \sin n \tau} \sum_{j=0}^{n-1}\left(k_{j}^{\prime} \sin (n-2 j) \tau-2 k_{j}^{\prime \prime} \cos 2 \tau \sin (n-2 j) \tau\right) \\
& -\frac{1}{2 \sin m \tau} \sum_{j=0}^{m-1}\left(l_{j}^{\prime} \sin (m-2 j) \tau-2 l_{j}^{\prime \prime} \cos 2 \tau \sin (m-2 j) \tau\right) \\
\phi_{2}(\tau) & =\frac{\cos 2 \tau}{2 \sin n \tau} \sum_{j=0}^{n-1}\left(k_{j}^{\prime} \sin (n-2 j) \tau-2 k_{j}^{\prime \prime} \cos 2 \tau \sin (n-2 j) \tau\right) \\
& -\frac{\cos 2 \tau}{2 \sin m \tau} \sum_{j=0}^{m-1}\left(l_{j}^{\prime} \sin (m-2 j) \tau-2 l_{j}^{\prime \prime} \cos 2 \tau \sin (m-2 j) \tau\right) \\
& -\frac{\sin 2 \tau}{2 \sin n \tau} \sum_{j=0}^{n-1}\left(k_{j}^{\prime} \cos (n-2 j) \tau-2 k_{j}^{\prime \prime} \cos 2 \tau \cos (n-2 j) \tau\right) \\
& +\frac{\sin 2 \tau}{2 \sin m \tau} \sum_{j=0}^{m-1}\left(l_{j}^{\prime} \cos (m-2 j) \tau-2 l_{j}^{\prime \prime} \cos 2 \tau \cos (m-2 j) \tau\right) .
\end{aligned}
$$

Writing $\phi_{j}(\tau)=p_{j}(\tau) / q_{j}(\tau), j=1,2$ as irreducible rational trigonometric polynomials, and following the method in [5], we only need to show that $p_{1}(\tau), p_{2}(\tau)$ are not identically zero. Expand $p_{1}(\tau), p_{2}(\tau)$ in terms of a finite sum of $\sin j \tau$ and/or $\cos j \tau$ terms. If we can show their highest frequency terms are not identically zero, from Lemma 2 in $[5], p_{1}(\tau), p_{2}(\tau)$ cannot be identically zero, as their highest frequency terms cannot be cancelled by finite sum of their lower frequency terms. So the lemma can be proved.

The highest frequency term $h_{1}(\tau)$ in $p_{1}(\tau)$ is

$$
h_{1}(\tau)=\left\{\begin{array}{cc}
\left(k_{0}^{\prime \prime}-l_{0}^{\prime \prime}\right) \sin (n+2) \tau & \text { if } m=n \\
\frac{1}{2}\left(k_{0}^{\prime \prime}-l_{0}^{\prime \prime}\right) \cos (m+n+2) \tau & \text { if } m \neq n
\end{array}\right.
$$

and the highest frequency term $h_{2}(\tau)$ in $p_{2}(\tau)$ is

$$
h_{2}(\tau)=\left\{\begin{array}{cc}
\left(k_{n-1}^{\prime \prime}-l_{n-1}^{\prime \prime}\right) \sin (-n-2) \tau & \text { if } m=n \\
-\frac{1}{2}\left(k_{n-1}^{\prime \prime}-l_{n-1}^{\prime \prime}\right) \cos (m+n+2) \tau & \text { if } m \neq n .
\end{array}\right.
$$

In case I then $k_{0}^{\prime \prime}=1, l_{0}^{\prime \prime}=-1$; conversely, in case II we must consider two sub-cases. The first of these has $z_{\mathbf{k}} \in A, z_{\mathbf{l}} \in B$,

$$
\begin{array}{lll}
f^{n-1}\left(z_{\mathbf{k}}\right)=f^{-1}\left(z_{\mathbf{k}}\right) \in A \cup B & \text { then } & k_{n-1}^{\prime \prime}=1, \\
f^{m-1}\left(z_{\mathbf{l}}\right)=f^{-1}\left(z_{\mathbf{l}}\right) \in C \cup D & \text { then } & l_{n-1}^{\prime \prime}=-1 ;
\end{array}
$$

if $z_{\mathbf{k}} \in C, z_{\mathbf{l}} \in D$,

$$
\begin{array}{cll}
f^{n-1}\left(z_{\mathbf{k}}\right)=f^{-1}\left(z_{\mathbf{k}}\right) \in C \cup D & \text { then } & k_{n-1}^{\prime \prime}=-1, \\
f^{m-1}\left(z_{\mathbf{l}}\right)=f^{-1}\left(z_{\mathbf{l}}\right) \in A \cup B & \text { then } & l_{n-1}^{\prime \prime}=1 .
\end{array}
$$

Therefore in all cases the highest frequency terms $h_{1}(\tau)$ and $h_{2}(\tau)$ are not identically zero and the lemma is proved.

We now present and prove the main result of this section. 
Theorem 1 There is a countable set of $\theta \in[\pi / 3,2 \pi / 3] \cup[4 \pi / 3,5 \pi / 3]$ for which there are any tangencies between periodic disks in the disk packing $\mathcal{C}$ induced by $f_{\theta}$ in (3). Therefore there is a full measure set of such $\theta$ such that the disk packing $\mathcal{C}$ has no tangencies.

Proof We prove this by showing that tangencies can only occur at isolated parameter values. If we consider the set of all possible periodic sequences of the four symbols $\{(1,1),(-1,1),(-1,-1),(1,-1)\}$, this is countable, as is the set of pairs of non-identical periodic sequences. For any such pair $(\mathbf{k}, \mathbf{l})$ Lemma 1 shows that there are at most a finite number of $\theta$ at which tangencies can occur. Hence the set of $\theta$ for which there are tangencies is at most countably infinite and the result follows.

\subsection{Some remarks}

As noted in [7] there is a natural equivalence for PWIs in terms of similarity. We have that the set $\operatorname{PWI}(n, m)$ of piecewise isometries of $n$ atoms that are convex $m$-gons has the structure of a union of manifolds of differing dimensions; these manifolds we refer to as classes of PWIs and their dimensions modulo similarity we refer to as perturbation dimensions.

The piecewise isometry $f$ induced by the $\Sigma-\Delta$ modulator has a quite high perturbation $\operatorname{dimension} \operatorname{dim}_{p}(f)=4$. Apart from the perturbation parameter $\theta$, the position of the two trapezia can be perturbed to be separated, see Figure 5(a).

By identifying points that are symmetrically related by a half-turn around the origin, the map $f$ can be reduced to the piecewise isometry $g$ illustrated in Figure 5(b). For this first return map, the only parameter that can be varied without introducing new atoms or new sides is the angle $\theta$.

Note that it is much easier to prove that the packing of invariant disks is tangent-free for the map $g$, as we only need to show the highest frequency term $h_{1}(t)$ in $p_{1}(t)$ is not identically zero (see Section 2.3).

We consider briefly now the map describing the $\Sigma-\Delta$ modulator with unity gain and non-zero constant input, i.e., in (1) $r=1$, and $x_{n}=a=$ constant $\neq 0$. In this case the piecewise isometry induced from the map can be written as:

$$
\left(\begin{array}{c}
w_{n+1} \\
y_{n+1}
\end{array}\right)=\left(\begin{array}{cc}
0 & 1 \\
-1 & 2 \cos \theta
\end{array}\right)\left(\begin{array}{c}
w_{n} \\
y_{n}
\end{array}\right)+\left(\begin{array}{c}
0 \\
a(2 \cos \theta-1)+\operatorname{sgn} w_{n}-2 \cos \theta \operatorname{sgn} y_{n}
\end{array}\right)
$$

Introduce $u_{n}=w_{n} / \sin \theta-y_{n} \cot \theta, v_{n}=y_{n}$ and denote $\mathbf{x}_{n}=\left[u_{n}, v_{n}\right]^{T}$ as before; then the piecewise isometry induced from the above map becomes

$$
\mathbf{x}_{n+1}=f\left(\mathbf{x}_{n}\right)=R \mathbf{x}_{n}+\left(\sigma\left(\mathbf{x}_{n}\right)+a(2 \cos \theta-1)\right)\left(\begin{array}{c}
-\cot \theta \\
1
\end{array}\right)
$$

where $\sigma\left(\mathbf{x}_{n}\right)=\operatorname{sgn}\left(u_{n} \sin \theta+v_{n} \cos \theta\right)-2 \cos \theta \operatorname{sgn} v_{n}$ and $R$ represents a pure clockwise rotation by $\theta$ (see Figure 6 ). The picture is similar to Figure 1 but the two trapezia here become asymmetrical.

Similarly to the zero input case, we can rewrite (10) in the complex form by

$$
f(z)=e^{-i \theta} z+W(\sigma(z)+2 a \cos \theta-a)
$$




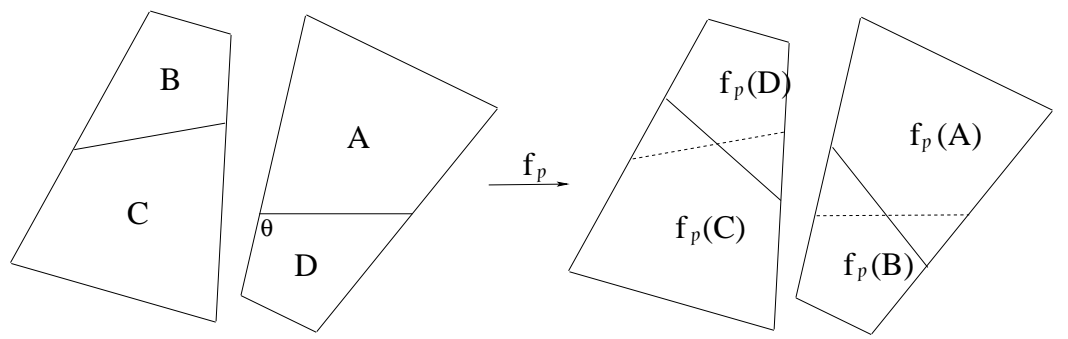

(a)

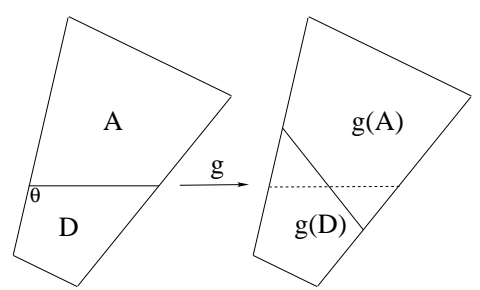

(b)

Figure 5: (a): A general perturbation of the $\Sigma-\Delta$ map $f$ that preserves the same number of quadrilateral atoms. (b): The piecewise isometry $g$ reduced from the $\Sigma-\Delta$ map $f$ by identifying points that are symmetrically related.

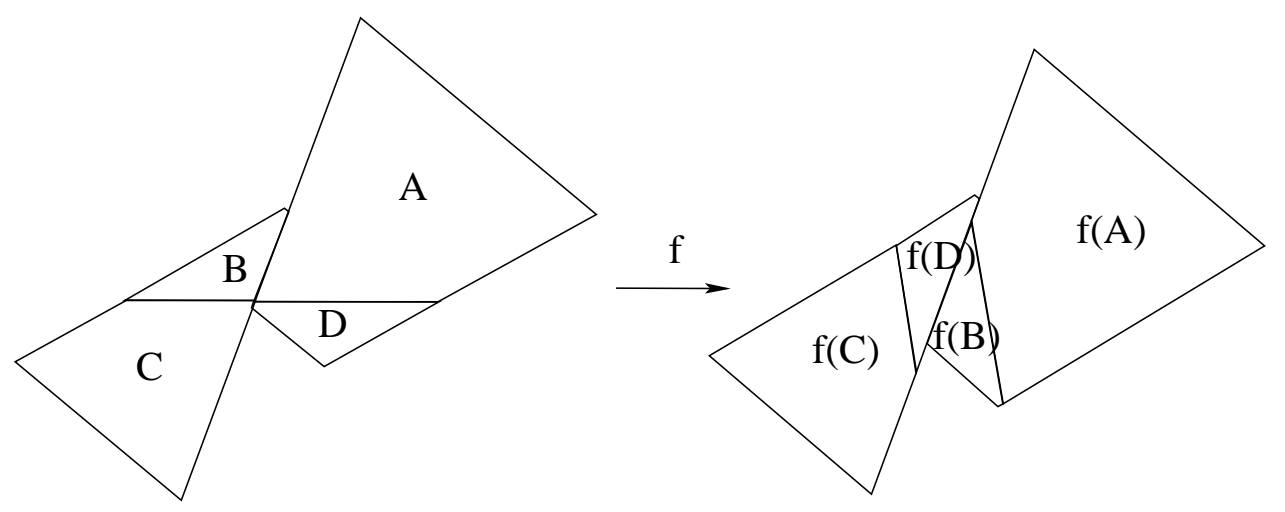

Figure 6: Invariant set for the bandpass $\Sigma-\Delta$ modulator map with constant input; $\theta=$ 1.9198 radians. 
where $W=-\frac{\omega}{\sin \theta}, \omega=e^{-i \theta}, \sigma(z)$ is the same as in (4). Again we assign each $A, B, C, D$ with a pair $k(z)=\left(k^{\prime}(z), k^{\prime \prime}(z)\right)$ which take the same values as (5). Then $f$ can be written as

$$
f(z)=\omega z+W k^{\prime}(z)-2 \cos \theta W k^{\prime \prime}(z)+2 a \cos \theta-a .
$$

By using the coding $k(z)=\left(k^{\prime}(z), k^{\prime \prime}(z)\right)$ we can parallel the discussion as in Section 2.2 and Section 2.3.

\section{Further examples: The Goetz map}

The proof of Theorem 1 relies on the fact that the centres of the disks can be found explicitly and are nontrivial analytic functions of the parameter. It is probable that such a proof can be adapted to show that there are rare tangencies for some more piecewise isometric maps, e.g. the affine maps of the form examined in [13, 15]; and maps derived from a kicked Hamiltonian system [19]. In Section 4 we will discuss further some more general families of piecewise isometries (such that the number of atoms and their faces are preserved; [7]) to which we are able to apply a similar method.

In [13] Goetz investigates a family of piecewise rotations of the plane about two centres $z_{0}, z_{1}$. We assume that the rotation angles $\theta$ are the same on each side. We write this Goetz map $T: \mathbf{C} \rightarrow \mathbf{C}$ in complex form:

$$
T(z)= \begin{cases}\omega\left(z-z_{0}\right)+z_{0} & \text { if } \operatorname{Re}(z)<0 \\ \omega\left(z-z_{1}\right)+z_{1} & \text { if } \operatorname{Re}(z) \geq 0\end{cases}
$$

where $\omega=e^{i \theta}$. In [13] it was shown that the dynamics of the map (11) may possess infinitely many periodic disks.

If we fix the left centre of rotation at $z_{0}=-1$, the family can be parameterized by the right centre $z_{1}=a+i b$. Denoting $P_{0}=\{z: \operatorname{Re}(z)<0\}, P_{1}=\{z: \operatorname{Re}(z) \geq 0\}$, the (11) becomes

$$
T(z)=\left\{\begin{array}{cc}
\omega z-(1-\omega) & \text { if } z \in P_{0} \\
\omega z+(a+i b)(1-\omega) & \text { if } z \in P_{1}
\end{array}\right.
$$

Let $\alpha(z)=\left(\alpha_{0}(z), \alpha_{1}(z)\right)$, where $\alpha_{i}(z)=\delta_{i j}$, for $z \in P_{j}, j=0,1$. Then we can use $\{(1,0),(0,1)\}$ as the coding, since we have

$$
T(z)=\omega z+(1-\omega)\left(-\alpha_{0}(z)+(a+i b) \alpha_{1}(z)\right)
$$

then for any admissible sequence $\left(k_{0} k_{1} \cdots k_{j} \cdots\right)$, where $k_{j}=\left(k_{j}^{\prime}, k_{j}^{\prime \prime}\right) \in\{(1,0),(0,1)\}$, there is a unique $z$ such that the $n$-th iterate of $T$ can be expressed as

$$
T^{n}(z)=\omega^{n} z+\sum_{j=0}^{n-1}(1-\omega) \omega^{j}\left(-k_{n-1-j}^{\prime}+(a+i b) k_{n-1-j}^{\prime \prime}\right) .
$$

So for a periodic point $z_{\mathbf{k}}$ of period $n$ with intinerary $z_{\mathbf{k}}=P\left(k_{0} k_{1} \cdots k_{n-1}\right)$, we have

$$
z_{\mathbf{k}}=\frac{1-\omega}{1-\omega^{n}} \sum_{j=0}^{n-1} \omega^{j}\left(-k_{n-1-j}^{\prime}+(a+i b) k_{n-1-j}^{\prime \prime}\right)
$$


If two disks with centres $z_{\mathbf{k}}, z_{\mathbf{l}}$ in different atoms are tangent, then

$$
\operatorname{Re}\left(\frac{z_{\mathbf{k}}-z_{\mathbf{l}}}{\cos \frac{\pi}{2}+i \sin \frac{\pi}{2}}\right)=0
$$

that is

$$
\operatorname{Im}\left(z_{\mathbf{k}}-z_{\mathbf{l}}\right)=0
$$

By making similar arguments to those in Section 2.3, one can show that (15) is not identically zero, so the tangencies between disks are rare, as indicated by numerical simulations; for example, Figure 6 in [13].

We note that this map is non-invertible and we show in the appendix that the planar kicked Hamiltonian map discussed by [19] may be viewed as a special case of the Goetz map.

\section{Discussion}

Although the models we consider are quite simple, we hope that this work may act as a guide to develop appropriate techniques for more complicated piecewise linear systems.

Restricting to planar PWIs, we believe that the tangent-free property is generic for all but the most trivial families of PWIs. In this section we try to point out the obstructions we have encountered in trying to make this statement more precise and hence provable.

Clearly, for any planar orientation preserving piecewise isometry (not necessarily invertible), as long as the atoms are convex, all periodically coded cells will be convex polygons. These will be disks if the rotation on return is by an irrational angle.

Supposing that the set of isometries is given by $f_{k}(z)=\omega_{k} z+w_{k}$ where $\omega_{k}=e^{i \theta_{k}}$, it is clear that given any periodic itinerary $\left\{k_{1}, \cdots, k_{n}\right\}$ one can obtain a unique expression for the location of a periodic orbit with this itinerary as long as

$$
\exp \left(i \sum_{j=1}^{n} \theta_{k_{j}}\right) \neq 1 \text {. }
$$

The first problem is this; such an inequality is satisfied if we assume that $\theta_{k}=\theta$ is constant and an irrational multiple of $\pi$ (or more generally, if $\theta_{k} \in \theta \mathbf{N}$ for some $\theta$ multiple of $\pi$ ). Hence for many of the one-parameter classes of PWIs that have irrational rotations in [7] we can assure this. For more general PWIs there may be parameter values where the inequality above is not satisfied.

The second problem is to show that given two periodic orbits $z_{\mathbf{k}}$ and $z_{\mathbf{l}}$, the distance between where their perpendiculars meet any discontinuity line (which will necessarily be an analytic function of the parameter $\omega$ ) is a non-trivial function of this parameter. In the proof for the $\Sigma-\Delta$ map, this requires a detailed argument; a more general result would presumably need to avoid this.

For a general PWI which is an irrational rearrangement, when $\theta \notin \pi \mathbb{Q}$, then an admissible periodic coding corresponds to a unique periodic point of the PWI. Hence, if one can determine all the admissible periodic codings, the periodic and quasi-periodic motion of the system then the invariant disk packing $\mathcal{C}$, is determined, and also the exceptional set $\mathcal{D}=\mathcal{C}^{c}$. 
It is a surprisingly difficult problem to identify the set of periodic admissible codings $[18,20]$. The only case with a relatively complete understanding is the case of theoverflow map with $\theta_{k}=\pi / 4$ [1], which is a rational angle. In fact, for a general piecewise isometry it is still not even known whether $\mathcal{C}$ has an infinite or finite number of connected components or even if it is non-empty.

\section{Acknowledgement}

We thank the EPSRC for support via grant GR/M36335.

\section{References}

[1] R. Adler, B. Kitchens and C. Tresser, Dynamics of nonergodic piecewise affine maps of the torus. Ergod. Th. ES Dynam. Sys. 21:959-999 (2001).

[2] P. Ashwin, Elliptic behaviour in the sawtooth standard map, Phys. Lett. A 232:409416 (1997).

[3] P. Ashwin, W. Chambers, W. G. and Petkov, Lossless digital filter overflow oscillations; approximation of invariant fractals. Int. J. Bifur. Chaos 7:2603-2610 (1997).

[4] P. Ashwin, J.H.B. Deane and X.-C. Fu, Dynamics of a bandpass sigma-delta modulator as a piecewise isometry, Proceedings, ISCAS'2001, (Proceedings of the IEEE International Symposium on Circuits and Systems, 2001), pp III-811 - III-814 (Sydney, Australia, 2001)

[5] P. Ashwin and X.-C. Fu, Tangencies in invariant disk packings for certain planar piecewise isometries are rare. Dynamical Systems 16:333-345 (2001).

[6] P. Ashwin and Xin-Chu Fu, On the dynamics of some nonhyperbolic area-preserving piecewise linear maps, to appear in Mathematics in Signal Processing (the volume of papers selected from those presented at the Fifth IMA Conference on Maths in Signal Processing held at Univ. of Warwick, December 2000), Oxford University Press IMA Conference Series.

[7] P. Ashwin and Xin-Chu Fu, On the geometry of planar piecewise isometries. Preprint, University of Exeter, 2001.

[8] A.C. Davies, Nonlinear oscillations and chaos from digital filter overflow. Phil. Trans. R. Soc. Lond. A 353:85-99 (1995).

[9] O. Feely, Nonlinear dynamics of bandpass sigma-delta modulation, Proceedings of NDES, Dublin, pp 33-36 (1995).

[10] O. Feely and D. Fitzgerald, Non-ideal and chaotic behaviour in bandpass sigma-delta modulators Proceedings of NDES 1996, Sevilla, Spain, pp 399-404 (1996);

[11] O. Feely and D. Fitzgerald, Bandpass sigma-delta modulation: an analysis from the perspective of nonlinear dynamics, Proceedings of ISCAS 1996, Atlanta, Ga, May 1996. 
[12] A.C. Davies, Periodic Non-linear Oscillations from Bandpass sigma-delta modulators', Proceedings of ISCAS 1996, Atlanta, Ga, pp. 469-472 (1996).

[13] A. Goetz, Perturbation of 8-attractors and births of satellite systems, Intl. J. Bifn. Chaos 8:1937 (1998).

[14] A. Goetz, Dynamics of a piecewise rotation. Continuous and Discrete Dynamical Systems 4:593-608 (1998).

[15] A. Goetz, Stability of cells in piecewise rotations and affine maps. Nonlinearity 14:205-219 (2000).

[16] A. Goetz, Dynamics of a piecewise isometries. Illinois J. Math. 44:465-478 (2000).

[17] E. Gutkin and H. Haydn, Topological entropy of generalized polygon exchanges. Bull. Amer. Math. Soc. 32:50-57 (1995).

[18] L. Kocarev, C.W. Wu and L.O. Chua, Complex behaviour in Digital filters with overflow nonlinearity: analytical results. IEEE Trans CAS-II 43:234-246 (1996).

[19] A.J. Scott, C.A. Holmes and G.J. Milburn, Hamiltonian mappings and circle packing phase spaces, Physica D 155:34-50 (2001).

[20] C.J. Vowden, PhD thesis, Department of Engineering, University of Warwick, UK (1999). 


\section{Appendix: Planar area preserving map derived from a kicked Hamiltonian system}

In [19] some area preserving maps derived from a kicked Hamiltonian system with planar, hyperbolic or spherical phase space structures which resemble circle packings are discussed. For the case of the planar phase space, they examine a Hamiltonian of the form

$$
H(x, p, t)=\frac{1}{2} \omega\left(x^{2}+p^{2}\right)+\mu|x| \sum_{n=-\infty}^{\infty} \delta(t-n),
$$

where $x, p$ and $t$ are position, momentum and time, respectively, and $\omega \in[0,2 \pi)$ and $\mu \geq 0$ are parameters. The mapping which takes $(x, p)$ from just before a kick to one period later is

$$
\left(\begin{array}{l}
x_{n+1} \\
p_{n+1}
\end{array}\right)=F\left(\begin{array}{l}
x_{n} \\
p_{n}
\end{array}\right)=\left(\begin{array}{cc}
\cos \omega & \sin \omega \\
-\sin \omega & \cos \omega
\end{array}\right)\left(\begin{array}{c}
x_{n} \\
p_{n}-\mu s_{n}
\end{array}\right)
$$

where $s_{n}=\operatorname{sgn} x_{n}$. Letting $z=x+i p$, we rewrite the map (16) in the complex form by

$$
F(z)=e^{-i \omega} z+W k(z)
$$

where $W=-\frac{i \omega}{\lambda}, \lambda=e^{i \omega}$, and

$$
k(z)=\left\{\begin{array}{rl}
1 & \text { if } \operatorname{Re}(z)>0 \\
0 & \text { if } \operatorname{Re}(z)=0 \\
-1 & \text { if } \operatorname{Re}(z)<0
\end{array} .\right.
$$

The map (17) is clearly equivalent to a special case of the Goetz map (11) for almost all initial conditions. 